\title{
A NONCONVERGENT ITERATIVE PROCESS
}

J. H. ROBERTS

1. Introduction. In [1] Mann and Wolf considered the integral equation

$$
y(t)=\int_{0}^{t} \frac{G[y(x)]}{[\pi(t-x)]^{1 / 2}} d x,
$$

where

(2) $G(y)$ is continuous and strictly decreasing for positive $y$, and

$$
G(1)=0 \text {. }
$$

They defined a sequence of functions $y_{0}(t), y_{1}(t), \ldots$ inductively as follows:

$$
y_{0}(t)=0, \quad y_{n+1}(t)=\int_{0}^{t} \frac{G\left[y_{n}^{*}(x)\right]}{[\pi(t-x)]^{1 / 2}} d x,
$$

where $y_{n}^{*}(x)=\min \left(y_{n}(x), 1\right)$. Under the additional assumption that $G(y)$ satisfies a Lipschitz condition on $[0,1]$ they proved that the sequence $y_{0}(t), y_{1}(t), \cdots$ converges to a bounded solution, ${ }^{1} y(t)$, of (1). Dr. Mann pointed out to me that it was not known whether or not the requirement of a Lipschitz condition was superfluous. The present paper resolves this uncertainty by giving an example of a function $G(y)$ satisfying (2) for which the corresponding sequence (3) does not converge. It also contains a positive result, to the effect that the sequence defined by (3) does converge to the solution $y(t)$ if, in addition to requirement (2), $G(y)$ is convex.

2. The counter example. The desired function $G(y)$ is defined as follows:

$$
\begin{array}{lrl}
G(y)=1-y & \text { for } 0 \leqq y \leqq 1 / 2 ; \\
G(y)=\left[1-(2 y-1)^{1 / 8}\right] / 2 & \text { for } 1 / 2<y .
\end{array}
$$

Let $G_{1}(y)=1-y$ for $y \geqq 0$, and let $z(t)$ be the bounded solution of

Presented to the Society, April 26, 1952; received by the editors September 30, 1952 and, in revised form, January 14, 1953.

1 It was shown in [2] that even in the absence of the Lipschitz condition equation (1) has a unique bounded solution $y(t)$, provided only that $G$ satisfies requirement (2). This solution $y(t)$ is strictly increasing and approaches the limit 1 as $t$ increases indefinitely. 


$$
z(t)=\int_{0}^{t} \frac{G_{1}[z(x)]}{[\pi(t-x)]^{1 / 2}} d x .
$$

Now, as was shown in $[1$, p. 168], $z(t)$ is continuous $(t \geqq 0)$ and $d z / d t$ is positive and decreasing for $t>0$. Thus a positive number $\alpha$ is uniquely determined by the requirement $z(\alpha)=1 / 2$. Let $k$ be $d z / d t$ evaluated at $2 \alpha$, and let $\beta$ be the smaller of $2 \alpha$ and $\alpha+4 \alpha^{3} k^{2}$. Then clearly

$$
z(t) \geqq 1 / 2+k(t-\alpha) \quad(\alpha \leqq t \leqq \beta) .
$$

LEMMA 1. If, for some $n, y_{n}(t) \geqq z(t)$ for $0 \leqq t \leqq \beta$, then over the same interval $y_{n+1}(t) \leqq \min (z(t), 1 / 2)$.

LEMMA 2. If, for some $n, y_{n}(t) \leqq \min (z(t), 1 / 2)$ for $0 \leqq t \leqq \beta$, then over the same interval $y_{n+1}(t) \geqq z(t)$.

Assuming that these lemmas are true, then $y_{2 r}(t) \leqq \min (z(t), 1 / 2)$, and $y_{2 r+1}(t) \geqq z(t)$, on $0 \leqq t \leqq \beta$. Then clearly the sequence $y_{0}(t)$, $y_{1}(t), \cdots$ does not converge for any $t$ between $\alpha$ and $\beta$ because $z(t)$ $>1 / 2$ over this range.

Proof of Lemma 1. Define $Y(t)$ as follows:

$$
Y(t)=\int_{0}^{t} \frac{G[z(x)]}{[\pi(t-x)]^{1 / 2}} d x .
$$

Since $y_{n}(x) \geqq z(x)$ for $0 \leqq x \leqq \beta$ and $G$ is a decreasing function, we have $y_{n+1}(t) \leqq Y(t)$. Now $Y(t)=z(t)$ for $0 \leqq t \leqq \alpha$; we shall show that $Y(t)$ $<1 / 2$ for $\alpha<t \leqq \beta$. Throughout the remainder of the proof $t$ will be a fixed number $\alpha+\Delta t, 0<\Delta t \leqq \beta-\alpha$. From (7) we have

$$
\begin{aligned}
Y(t)-Y(\alpha)= & \int_{\alpha}^{\alpha+\Delta t} \frac{G[z(x)]}{[\pi(\alpha+\Delta t-x)]^{1 / 2}} d x \\
& -\int_{0}^{\alpha} G[z(x)]\left[\frac{1}{[\pi(\alpha-x)]^{1 / 2}}\right. \\
& \left.-\frac{1}{[\pi(\alpha+\Delta t-x)]^{1 / 2}}\right] d x, \\
= & \text { Gain - Loss, say. }
\end{aligned}
$$

Now $G[z(x)] \geqq 1 / 2$ over $0 \leqq x \leqq \alpha$, and integration gives

$$
\text { Loss } \geqq \pi^{-1 / 2}\left(\alpha^{1 / 2}-(\alpha+\Delta t)^{1 / 2}+(\Delta t)^{1 / 2}\right) \text {. }
$$

To get an upper bound on the gain in (8) we first use (6) and (4), 
and find that $G[z(x)] \leqq\left(1-[2 k(x-\alpha)]^{1 / 8}\right) / 2=f(x)$, say. Next we replace $f(x)$ by the linear function $F(x)$ determined to equal $f(x)$ at $x=\alpha$ and at $x=\alpha+\Delta t$. Since $f(x)$ is convex we clearly have $f(x) \leqq F(x)(\alpha \leqq x$ $\leqq \alpha+\Delta t)$, and thus

(10) $G[z(x)] \leqq F(x)=1 / 2-m(x-\alpha)$, where $m=(\Delta t)^{-2 / 3}(2 k)^{1 / 8} / 2$.

Substituting for $G[z(x)]$ in the first integral of (8) and performing the integration gives

$$
\text { Gain } \leqq \pi^{-1 / 2}\left[(\Delta t)^{1 / 2}-(4 m / 3)(\Delta t)^{8 / 2}\right] .
$$

Then, from (11) and (9),

$$
\begin{aligned}
\text { Gain - Loss } & \leqq \pi^{-1 / 2}\left((\alpha+\Delta t)^{1 / 2}-\alpha^{1 / 2}-(4 m / 3)(\Delta t)^{3 / 2}\right) \\
& \leqq \pi^{-1 / 2}\left[\Delta t\left(\alpha^{-1 / 2} / 2\right)-(4 m / 3)(\Delta t)^{3 / 2}\right] \\
& =\left(\pi^{-1 / 2} \Delta t\right)\left[\left(\alpha^{-1 / 2} / 2\right)-(4 m / 3)(\Delta t)^{1 / 2}\right]
\end{aligned}
$$

In the last member of (12) replace $m$ by its value (see (10)), and replace $\Delta t$ (in the second factor) by its upper bound, $4 \alpha^{8} k^{2}$. This gives

$$
\text { Gain - Loss } \leqq \pi^{-1 / 2} \Delta t\left[\alpha^{-1 / 2} / 2-2 \alpha^{-1 / 2} / 3\right]<0 .
$$

This completes the proof of Lemma 1.

Proof of Lemma 2. Now $G(y)=G_{1}(y)$ for $0 \leqq y \leqq 1 / 2$, so under the hypothesis that $y_{n}(t) \leqq \min (z(t), 1 / 2)$ we know that

$$
G\left[y_{n}(x)\right]=G_{1}\left[y_{n}(x)\right] \quad \text { for } 0 \leqq x \leqq \beta .
$$

Then over this range

$$
y_{n+1}(t)=\int_{0}^{t} \frac{G_{1}\left[y_{n}(x)\right]}{[\pi(t-x)]^{1 / 2}} d x \geqq \int_{0}^{t} \frac{G_{1}}{[\pi(t-x)]^{1 / 2}} d x=z(t) .
$$

With this proof of Lemma 2 our discussion of the counterexample is complete.

3. The theorem. If $G(y)$ satisfies (2) and in addition is convex for $0 \leqq y \leqq 1$, then the sequence $y_{0}(t), y_{1}(t), \cdots$ given by (3) converges to the solution $y(t)$ of (1).

Proof. Now $y_{0}(t)=0$ and $y_{1}(t)=2 G(0)(t / \pi)^{1 / 2}$. Define positive numbers $d$ and $c$ by the respective requirements

$$
G(d)=3 G(0) / 4, \quad y_{1}(c)=d .
$$

We first prove the conclusion of the theorem for $t$ restricted to the interval $[0, c]$. 
From the convexity of $G(y)$ we see that for any $r_{1}$ and $r_{2}$ between 0 and $1\left(r_{1} \neq r_{2}\right)$ we have

$$
\left|\frac{G\left(r_{1}\right)-G\left(r_{2}\right)}{r_{1}-r_{2}}\right| \leqq \frac{G(0)-G\left(r_{1}\right)}{r_{1}} .
$$

From our choice of $d$ and $c$ and the fact that $y_{n}(t) \leqq y_{1}(t)$ for all $n$ we see from (2) that $G\left[y_{n}(t)\right] \geqq 3 G(0) / 4$ for $0 \leqq t \leqq c$. Then

$$
y_{n}(t) \geqq(3 / 4) y_{1}(t)=(3 / 2) G(0)(t / \pi)^{1 / 2} \text {. }
$$

Let $\Delta_{n}=\max \left|y_{n}(t)-y_{n-1}(t)\right|$ for $0 \leqq t \leqq c$. Thus

$$
\begin{aligned}
\left|y_{n+1}(t)-y_{n}(t)\right| & =\int_{0}^{t} \frac{\left|G\left[y_{n}(x)\right]-G\left[y_{n-1}(x)\right]\right|}{[\pi(t-x)]^{1 / 2}} d x \\
& \leqq \int_{0}^{t} \frac{\left(G(0)-G\left[y_{n}(x)\right]\right) \cdot\left|y_{n}(x)-y_{n-1}(x)\right|}{y_{n}(x)[\pi(t-x)]^{1 / 2}} d x \\
& \leqq \int_{0}^{t} \frac{[G(0) / 4] \Delta_{n}}{(3 / 2) G(0)(x / \pi)^{1 / 2}[\pi(t-x)]^{1 / 2}} d x \\
& =\frac{\Delta_{n}}{6} \int_{0}^{t} \frac{d x}{[x(t-x)]^{1 / 2}}=(\pi / 6) \Delta_{n} .
\end{aligned}
$$

(In the above we first use (3), then (15), and then (14) and (16). The two final equalities are obvious.) Thus $\left|y_{n+1}(t)-y_{n}(t)\right| \leqq \Delta_{n+1}$ $\leqq \Delta_{n}(\pi / 6) \leqq(\pi / 6)^{n}$, for $0 \leqq t \leqq c$. This proves the convergence on the interval $[0, c]$.

Suppose the theorem is false and that on some interval $[0, T]$ the sequence $y_{0}(t), y_{1}(t), \cdots$ does not converge. Now for every $t, y_{0}(t)$ $\leqq y_{2}(t) \leqq \cdots \leqq y(t) \leqq \cdots \leqq y_{3}(t) \leqq y_{1}(t)$. Therefore the $y$ 's of even subscript converge to a continuous limit function $Y_{1}(t)$ and the $y$ 's of odd subscript converge to a continuous limit function $Y_{2}(t)$, and $Y_{1}(t) \leqq y(t) \leqq Y_{2}(t)$. It is furthermore clear that the substitution of $Y_{1}(x)$ [respectively $Y_{2}(x)$ ] for $y(x)$ under the integral sign in (1) gives $Y_{2}(t)$ [respectively $Y_{1}(t)$ ] in place of $y(t)$. The convergence of $y_{0}(t)$, $y_{1}(t), \cdots$ on $[0, c]$ implies that $Y_{1}(t)=Y_{2}(t)$ for $0 \leqq t \leqq c$. Let $e$ be the greatest number such that $Y_{1}(t)=Y_{2}(t)$ for $0 \leqq t \leqq e$. Then $c \leqq e<T$.

Since $Y_{1}$ has a positive minimum value on $[e, T]$ it follows from the hypotheses on $G$ that there exists a positive $k$ such that for any $x$ on $[e, T], G\left[Y_{1}(x)\right]-G\left[Y_{2}(x)\right] \leqq k\left|Y_{1}(x)-Y_{2}(x)\right|$. Choose a fixed $t$ $(e<t<T)$ so that (a) $2 k[(t-e) / \pi]^{1 / 2}<1$ and (b) $\left|Y_{1}(x)-Y_{2}(x)\right|$ $\leqq\left|Y_{1}(t)-Y_{2}(t)\right|$ for $e \leqq x \leqq t$. Then 


$$
\begin{aligned}
\left|Y_{2}(t)-Y_{1}(t)\right| & =\int_{0}^{t} \frac{G\left[Y_{1}(x)\right]-G\left[Y_{2}(x)\right]}{[\pi(t-x)]^{1 / 2}} d x \\
& \leqq \int_{0}^{t} \frac{k\left|Y_{1}(x)-Y_{2}(x)\right|}{[\pi(t-x)]^{1 / 2}} d x \\
& \leqq k\left|Y_{1}(t)-Y_{2}(t)\right| \int_{0}^{t} \frac{d x}{[\pi(t-x)]^{1 / 2}} \\
& =2 k[(t-e) / \pi]^{1 / 2} \cdot\left|Y_{1}(t)-Y_{2}(t)\right| \\
& <Y_{2}(t)-Y_{1}(t) .
\end{aligned}
$$

Thus the assumption that the theorem is false has led to a contradiction.

\section{REFERENCES}

1. W. Robert Mann and Frantisek Wolf, Heat transfer between solids and gasses under nonlinear boundary conditions, Quarterly of Applied Mathematics vol. 9 (1951) pp. 163-184.

2. J. H. Roberts and W. R. Mann, On a certain nonlinear integral equation of Volterra type, Pacific Journal of Mathematics vol. 1 (1951) pp. 431-445.

DUKR UNIVERSITY 